\title{
Novel bioinformatics approach to investigate quantitative phenotype-genotype associations in neuroimaging studies
}

$4 \quad$ Sejal Patel $^{1,2 *}$, Min Tae M. Park ${ }^{3,4}$, The Alzheimer's Disease Neuroimaging Initiative ${ }^{\mathrm{a}}$, M.

5 Mallar Chakravarty ${ }^{3,5}$, Jo Knight ${ }^{1,2,6,7}$

$6{ }^{1}$ Campbell Family Mental Health Research Institute, Centre for Addiction and Mental Health,

7 Toronto, ON, Canada

$8 \quad{ }^{2}$ Institute of Medical Science, University of Toronto, Toronto, ON, Canada

$9{ }^{3}$ Cerebral Imaging Centre, Douglas Mental Health University Institute, McGill University, Verdun, 10 QC, Canada

${ }^{4}$ Schulich School of Medicine and Dentistry, Western University, London, ON, Canada

${ }^{5}$ Department of Psychiatry, McGill University, Montreal, QC, Canada

${ }^{6}$ Department of Psychiatry, University of Toronto, Toronto, ON, Canada

${ }^{7}$ Dalla Lana School of Public Health, Biostatistics Division, University of Toronto, Toronto, ON, Canada

\section{* Correspondence:}

Sejal Patel

Campbell Family Mental Health Research Institute

Centre for Addiction and Mental Health

250 College Street

Toronto, ON, M5T 1R8, Canada

Sejal.Patel@camh.ca

M. Mallar Chakravarty and Jo Knight are co-senior authors.

${ }^{\text {a }}$ Data used in preparation of this article were obtained from the Alzheimer's Disease Neuroimaging Initiative (ADNI) database (adni.loni.usc.edu). As such, the investigators within the ADNI contributed to the design and implementation of ADNI and/or provided data but did not participate in analysis or writing of this report. A complete listing of ADNI investigators can be found at: http://adni.loni.usc.edu/wp-content/uploads/how_to_apply/ADNI_Acknowledgement_List.pdf.

Keywords: Magnetic resonance imaging (MRI), Genome Wide Association Study (GWAS), Gene Ontology network, Alzheimer's disease (AD), Stratified false discovery rate (sFDR), Imaging genetics

Abstract

Imaging genetics is an emerging field in which the association between genes and neuroimagingbased quantitative phenotypes are used to explore the functional role of genes in neuroanatomy and neurophysiology in the context of healthy function and neuropsychiatric disorders. The main obstacle for researchers in the field is the high dimensionality of the data in both the imaging phenotypes and the genetic variants commonly typed. In this article, we develop a novel method that utilizes Gene Ontology, an online database, to select and prioritize certain genes, employing a stratified false discovery rate (sFDR) approach to investigate their associations with imaging phenotypes. sFDR has the potential to increase power in genome wide association studies (GWAS), and is quickly gaining 
Bioinformatics with quantitative phenotype-genotype associations

traction as a method for multiple testing correction. Our novel approach addresses both the pressing need in genetic research to move beyond candidate gene studies, while not being overburdened with a loss of power due to multiple testing. As an example of our methodology, we perform a GWAS of hippocampal volume using the Alzheimer’s Disease Neuroimaging Initiative sample.

\section{Introduction}

Imaging genetics is a burgeoning field that seeks to understand the association of neuroimagingbased phenotypes, such as structural, functional (Thompson et al., 2010) and diffusion imaging-based metrics, (Patel et al., 2010) with genetic variations. Candidate gene studies were initially the method of choice for understanding gene function in humans, and successfully identified genes involved in Mendelian diseases; however, such studies have had less success for complex genetic disorders, with many novel findings failing to replicate in further studies (Hirschhorn et al., 2002). Reasons for such failures include a lack of power to identify the small effect sizes typically involved in complex traits, as well as a lack of knowledge about which genes are appropriate to study (Tabor et al., 2002; Ioannidis, 2005). Around 2007, genome-wide association studies (GWAS) began to make inroads as an efficient method for identifying variants associated with complex disease. In this approach, approximately one million single nucleotide polymorphisms (SNPs) across the whole genome are interrogated simultaneously, hypothesis-free (Wellcome_Trust_Case_Control_Consortium, 2007). However, due to the large burden of multiple testing correction in a GWAS, a p-value of $5 \times 10^{-8}$ or less, roughly equivalent to a $\mathrm{p}=0.05$ after Bonferroni correction for half a million independent variants, is generally required for a SNP to be recognized as significantly associated with a trait (Dudbridge and Gusnanto, 2008). Given the polygenic nature of complex traits and low effect sizes associated with these traits, large sample sizes are required to achieve adequate statistical power. Recently, a large imaging genetics study named ENIGMA (Enhancing NeuroImaging Genetics through Meta-Analysis) was undertaken, in which 21,000 subjects were included in a GWAS in order to identify genetic variants with association to hippocampal volume (Stein et al., 2012). While this study was a landmark demonstration for the use of imaging genetics techniques to investigate brain structures, it is not plausible for individual investigators to obtain such large sample sizes for their studies.

Various approaches have been described to reduce the multiple testing burden for large scale GWAS. One such approach is to control for the false discovery rate (FDR), rather than the family-wise error rate (FWER) (Benjamini and Hochberg, 1995). Where the family-wise error rate identifies the probability of one type 1 error from the total tested hypotheses, FDR calculates the proportion of expected type 1 errors. Stratified false discovery rate (sFDR) is an extension of the FDR control approach, where the false discovery rate is controlled in distinct subsets (strata) of the data, one or more of which are believed to have a higher prior probability of being associated with the trait of interest. Strata are defined based on prior information such as linkage analysis, candidate gene studies, or biological pathways (Sun et al., 2006; Sun et al., 2012). An example of this approach by Sun et al. (2012) investigates the susceptibility to meconium ileus (severe intestinal obstruction) in individuals with cystic fibrosis by prioritizing a set of genes involved in the apical plasma membrane. In this article, Sun et al. (2012) selected strata defined by Gene Ontology (GO) terms. GO is a biomedical ontology database, which contains structured vocabulary terms known as GO terms designed to describe protein function (Ashburner et al., 2000). In more complex traits, this approach may not be refined enough for the proper stratification of data in sFDR. In the case of Alzheimer's disease (AD), for example, a vast number of SNPs would be selected to have prior association, and this may reduce the value of stratification. We present a novel method that employs information from 
previous studies alongside GO in order to create a refined list of relevant genes to be analyzed in the sFDR framework.

We demonstrate our method's efficacy by investigating the association between genetic variants and hippocampal volume in the Alzheimer's Disease Neuroimaging Initiative (ADNI1) dataset; sFDR is performed to stratify genes of particular interest to $\mathrm{AD}$. The dominant symptom of AD is dementia, where memory, reasoning, and thinking are all impaired. The hippocampus plays a key role in cognitive functioning, influencing processes such as learning and the ability to make new memories (Braskie et al., 2013). Further, in considering the neurodegeneration of medial temporal lobe structures, the changes in hippocampal structure are considered to be one of the strongest quantitative phenotypes associated with AD and can often be used to predict cognitive decline in AD patients (Braskie et al., 2013).

This article presents a novel, systematic method to determine the optimal stratification of SNPs for sFDR analysis. We employed GO alongside previous GWAS findings, and applied our method to the ADNI1 dataset. Our method reduces the multiple testing correction burden with the potential to discover novel biomarkers in imaging genetics. Useful not only for new genetic studies, our tool is highly applicable to mining already existing GWAS data and improving the integration of publically available bioinformatics resources such as GO with imaging genetics studies.

\section{$107 \quad 2 . \quad$ Materials and Methods}

\section{Box 1}

\section{Gene Ontology}

Gene Ontology (http://geneontology.org/) is a publically available, free, ontology database that describes protein function (Ashburner et al., 2000). Gene products - proteins - are classified and grouped in three main ontologies: cellular components (CC) where the protein is located within subcellular compartments, molecular functions (MF) indicates the specific function of the gene is carried out in normal conditions and biological processes (BP) which describes the processes a protein is involved in (e.g.: neurogenesis). The ontology follows a hierarchical order and there are defined relationships between the GO terms. In the ontology structure, terms at the top represent general or broad concepts, whereas terms near the bottom represent more detailed processes.

Therefore if a term has terms subordinate to it, it is referred to as a 'parent' term. Similarly, if a term has other terms superior to it, then it is referred to as a 'child' term. Both manual and automatic annotations of proteins are available in the GO database. Automatic annotations are inferred from electronic annotations and are not manually reviewed by a curator. In manual annotations, a curator reviews primary articles to generate annotations, and each annotation is based on experimental data referenced to a PubMed ID. The documentation for manual curation can be found at http://geneontology.org/page/annotation, and an example of annotations created by the authors can be found in the Alzheimer's University of Toronto dataset at http://www.ebi.ac.uk/QuickGO/GAnnotation?source=Alzheimers_University_of_Toronto Quick GO (http://www.ebi.ac.uk/QuickGO/) is a web based tool used to extract data from the GO database.

\section{Cytoscape}

Cytoscape is an open source software platform visualization tool used to integrate data into complex networks of molecular interaction and biological pathways ((Saito et al., 2012), 
Bioinformatics with quantitative phenotype-genotype associations

http://www.cytoscape.org/). See Figure 4 as an example of a biological network.

\subsection{Selecting Priority List of Genes}

111 Below, we detail how we assembled a list of priority genes, derived comprehensive gene networks

112 based on these so-called "seed" genes, and pruned these networks appropriately. Our priority SNPs were selected from genes involved in biological systems associated with AD. Figure 1 outlines the entire process followed, including SNP selection (Figure 1A) and the preparation and subsequent analysis of the genetic and imaging data (Figure 1B).

Step 1: Twenty-one hits from a previous meta-analysis of AD GWAS signals were used as a starting point to identify top gene hits (Lambert et al., 2013). In addition we added amyloid precursor protein (APP) (Goate et al., 1991), Presenilin-1 (PSEN1) and Presenilin-2 (PSEN2) (Cruts et al., 1998) to our gene list based on association with higher risk of developing early onset of AD. Furthermore rare variants within these gene regions also increase the risk of late onset AD (Cruchaga et al., 2012) .

Step 2: Gene Ontology (GO) (refer to Box 1) was used to group genes, and subsequently to derive common biological process networks using a three step process detailed below.

Firstly, the biological process (BP) ontology dataset within GO was examined using Quick GO (refer to Box 1) in order to identify all BP terms associated with the genes under investigation, hereafter called originally selected GO terms (OGO terms). No restrictions were given on the type of evidence codes used for the annotation of the OGO terms. Secondly, similar OGO terms were grouped together to form key BP domain categories. Specifically, they were grouped based on common parent terms, which were higher up in the hierarchal ontology. Thirdly, common biological processes were identified based on the frequency of occurrence of previously associated genes. Only OGO terms associated with these common processes are carried forward to the next step.

Based on the outcome of the three steps outlined above, we grouped all child terms that derived from parent terms in the domains of synaptic function, neuroanatomical structure development, and neurogenesis. These three parent terms were found to be under the common network of "nervous system development and synaptic transmission”, which was identified as a common biological process. GO terms that fell under the network "nervous system development and synaptic transmission” were the fourth child term from the BP parent GO term. Refer to Box 1 under Gene Ontology section for child and parent terminology. In order to benchmark our approach in the selection of common biological processes, INRICH (Lee et al., 2012) was used as an alternative, objective, method to derive the common biological process domains. However, no significant results were identified to take forward to sFDR. The INRICH process is defined in the supplementary text.

Step 3: Cytoscape 2.8 (refer to Box 1) was used to visualize the biological process network "nervous system development and synaptic transmission”, and parent GO terms from the OGO terms were extracted to contextualize this network. As expected, the networks were overly complex and contained much extraneous information. To remedy this, an algorithm was developed to effectively reduce redundant data in order to create an effectively "pruned" network. This is accomplished by using building and pruning techniques based on the relationships of OGO terms. Figures 2-4 demonstrate different stages of this algorithm with the OGO terms in green boxes. Figure 2 shows a subsection of GO terms in the complete "nervous system development and synaptic transmission" network before pruning of the data. Figures 3A to 3D display how specific criteria were used to 
remove non-targeted GO terms. Figure 4 shows the final pruned data of the nervous system development and synaptic transmission network.

The following criteria were used to select the child and parent GO terms.

a. When extracting the ontology of the OGO terms using Cytoscape, child terms are automatically selected. Therefore, to simplify the ontology networks, child terms were removed. Orange terms in Figure 3A represent extra child terms of the OGO terms, which are not needed in the network. For example the GO term 'axonogenesis' has two child terms 'collateral sprouting' and 'peripheral nervous system neuron axonogenesis'. These are not necessary because the genes from step 1 have not been associated with these GO terms. (Figure 3A)

b. If more than one parent term is identified for an OGO term, then a common parent term, which is shared by most of the OGO terms, is chosen. As an example, the term 'axonogenesis' has two parent terms, namely, 'neuron projection morphogenesis' and 'cell morphogenesis involved in neuron differentiation'. In Figure 3B the term 'neuron projection morphogenesis', displayed in a pink box, is removed because the alternate parent term, 'cell morphogenesis involved in neuron differentiation', is a parent term to both the selected GO terms ‘dendrite morphogenesis' and 'axonogenesis'.

c. A positive or negative regulation child term will have two types of parents. As an example, we will investigate the term 'negative regulation of axonogenesis'. The first parent will be the term it regulates ('axongenesis') and the second parent would likely be a term that has 'regulation' as a key word in the term name, for example, 'regulation of axonogenesis' could be a candidate. Therefore the parent term that is regulated was selected, in this case the term 'axonogenesis', and the parent term that regulates a biological process but does not specify positive or negative regulation ('regulation of axonogenesis') is removed - shown in a yellow box -- because the child term will be more specific in terms of explaining how it is regulating the parent term (eg. negative regulation of axonogenesis), Figure 3C.

Step 4: Quick GO was used to extract all the genes that are associated to the OGO terms (as displayed in Figure 4 in green boxes) in the pruned "nervous system development and synaptic transmission" network. SNPs from these genes were extracted from the ADNI1 dataset using a reference file containing the start and end positions of the transcribed gene portion according to the Homo sapiens build 37 protein and coding genes from National Center for Biotechnology Information (NCBI). This list of SNPs formed the priority list for sFDR.

\subsection{ADNI Imaging Data}

\subsubsection{ADNI Data}

GWAS data and magnetic resonance imaging (MRI) neuroimaging data was obtained from the Alzheimer's Disease Neuroimaging Initiative (ADNI). Established in 2003 to facilitate the development of methods for biomarker investigation in order to enable detection of Alzheimer's disease (AD) at earlier stages, ADNI is a partnership between the National Institute on Aging, the National Institute of Biomedical Imaging and Bioengineering, the Food and Drug Administration, private pharmaceutical companies, and nonprofit organizations (http://adni.loni.usc.edu/ ; Michael W. Weiner, Principal Investigator). The ADNI database contains different information including neuroimaging, clinical, and genome-wide SNPs data. According to the ADNI protocol, subjects are diagnosed as cognitively normal (CN), mild cognitive impairment (MCI), or Alzheimer's disease (AD), based on the severity of their condition, and are recruited from Canada and the United States. 
Bioinformatics with quantitative phenotype-genotype associations

We used the ADNI1 dataset “ADNI1: Complete 1Yr 1.5T” (Wyman et al., 2013). 1.5T scanners (General Electric Healthcare, Philips Medical System or Siemens Medical Solutions) were used with the protocol described by (Jack et al., 2008). Before quality control (QC), 817 Caucasian 1.5T MRI subject scans were obtained from the ADNI1 database. Of the 817 subjects, 757 had GWAS data and 662 passed quality control. Figure 1B shows the overall steps taken to process the ADNI 1 MRI and GWAS data.

\subsubsection{Hippocampal Segmentation}

Hippocampal segmentation was carried out in all 662 samples with GWAS data, using a modified multi-atlas algorithm known as the Multiple Automatically Generated Templates (MAGeT-Brain) algorithm (Chakravarty et al., 2013; Pipitone et al., 2014). The MAGeT Brain algorithm overcomes the limitations of model-based segmentation techniques, and avoids the requirement for larger atlas libraries typically required in more traditional multi-atlas segmentation strategies (Heckemann et al., 2006; Collins and Pruessner, 2010) by bootstrapping the segmentation procedure using data from the participants being analyzed. The segmentation procedure consists of three steps. First, five highresolution MRI atlases developed by our group were used as inputs (Winterburn et al., 2013) and are used to automatically generate a "template library" based on a subset of the ADNI1 dataset using a model based segmentation procedure. For the purposes of this work we used a subset of subjects consisting of $7 \mathrm{AD}, 7 \mathrm{MCI}$ and $7 \mathrm{CN}$ subjects evenly distributed across an age range of 58-90 to model the anatomical variability across the ADNI1 dataset. Model-based segmentation is used to segment each of the subjects in the template library leading to a total of 5 candidate segmentations per subject. The next step proceeds much like a regular multi-atlas segmentation strategy, where each subject is nonlinearly matched to each of the subjects in the template library, yielding 105 (5 atlases $\times 21$ templates) candidate segmentations for each subject. The last step is a voxel voting technique where a label at each voxel that is most frequently occurring is used for the final segmentation (Collins and Pruessner, 2010). All resultant segmentations were manually inspected by an expert rater and only those segmentations passing quality control were used in the analysis. Images not successfully segmented by the MAGeT Brain algorithm were segmented manually for use. All input atlases (http://cobralab.ca/atlases/Hippocampus.html) and source code for MAGeT-Brain are freely available online (https://github.com/CobraLab/MAGeTbrain). Nonlinear transformations were estimated using the ANTs algorithm (Avants et al., 2008) and image processing steps were carried out using the MINC toolbox (http://www.bic.mni.mcgill.ca/ServicesSoftware/ServicesSoftwareMincToolKit).

\subsection{ADNI1 Genetic Data}

\subsubsection{Genetic Data Quality Control}

Quality control (QC) was performed on the ADNI 1 GWAS data (N=757) using PLINK (version 1.07, http://pngu.mgh.harvard.edu/ purcell/plink/ (Purcell et al., 2007)). In addition R (http://www.rproject.org/ ) was used to visualize the results. Individuals with discordant sex information, high level of missing data (> 2\%) and heterozygosity rates greater than 3 standard deviations from the mean were removed from the sample. One of each pair of individuals displaying a high level of pairwise identity by descent (IBD $>0.185$ ) were also removed. In addition, SNPs with minor allele frequency (MAF) $<1 \%$ and Hardy-Weinberg equilibrium ( $<1 \times 10^{-7}$ ) were removed. After QC, 662 individuals remained in the analysis set. Multidimensional scaling (MDS) was performed in PLINK using HapMap3 (Altshuler et al., 2010) as a reference panel. When the population is compared with the CEU (CEPH - Utah residents with ancestry from northern and western Europe), YRI (Yoruba in Ibadan, Nigeria), JPT (Japanese in Tokyo, Japan), TSI (Tuscans in Italy) and CHB (Han Chinese in Beijing, China) ancestry, the sample clustered around CEU and TSI sample. MDS was subsequently 
Bioinformatics with quantitative phenotype-genotype associations

carried out with the ADNI1, CEU, TSI and Jewish ancestry samples and aligned completely with the later three samples (Supplementary Figure S1). The Jewish ancestry sample was made available by Mark Silverberg.

\subsubsection{Data Preparation, Pre-Phasing and Imputation}

The GWAS data was based on UCSC, (University of California, Santa Cruz) build 36 reference (Lander et al., 2001), and the liftover tool available from the NCBI (http://genome.ucsc.edu/cgibin/hgLiftOver) was used to convert each SNP location to build 37. SHAPEIT 2.0 ((Delaneau et al., 2012), https://mathgen.stats.ox.ac.uk/genetics_software/shapeit/shapeit.html) was used to pre-phase the haplotypes of the GWAS data after QC. Imputation was performed on the pre-phased data using Impute2 ((Marchini et al., 2007), https://mathgen.stats.ox.ac.uk/impute/impute_v2.html) for the autosomal chromosomes with the 1000 Genome (March 2012) data as a reference. SNPs with info values of equal and greater than 0.5 and MAF $>0.05$ were retained for analysis.

\subsubsection{Association of Hippocampal Volume with GWAS Data}

SNPTEST 2.5 ((Marchini et al., 2007),

https://mathgen.stats.ox.ac.uk/genetics_software/snptest/snptest.html) was used to examine associations between hippocampal volumes with both imputed and genotyped SNPs. Covariates used in the analysis were gender, age, first dimension from MDS to control for population structure, baseline diagnoses (CN, MCI, or AD), APOE status because APOE e4 carriers have a higher risk of developing AD (Farrer et al., 1997) and intracranial volume to correct for variation in brain sizes within individuals in the sample. Three phenotypes were investigated: left hippocampal volume, right hippocampal volume, and mean (of the left and right) hippocampal volume. Frequentist association testing was undertaken for each phenotype, with a 'method' option in place to control for genotype uncertainty in the association test.

\subsection{Stratification of SNPs}

Fixed FDR strategies are used to control FDR in a group of tests. In sFDR, SNP p-values from the association analysis are grouped into distinct strata, one or more of which are believed to have a higher prior probability of being associated with the trait of interest (Sun et al., 2006). The association p-values of each SNP are transformed to q-values and FDR is controlled separately within each strata. To control the FDR at a given level $-5 \%$ in this analysis - the null hypothesis is rejected when tests have a q-value equal to or less than the specified threshold (0.05). This method increases the power to identify true associations if one of the strata is enriched with associated variants. When the strata aren't enriched, the method is still robust. Two SNP strata were formed in our data. All SNPs in the genes associated to the OGO terms (Figure 4) from the pruned "nervous system development and synaptic transmission" network formed one, high priority, strata (249,001 SNPs), and all the remaining SNPs formed the other (5,457,557 SNPs) in our non-priority stratum. Association p-values from SNPTEST were merged with each corresponding SNPs in each strata (priority and non-priority list) for sFDR. A Perl script was used to analyze priority and non-priority SNPs (http://www.utstat.toronto.edu/sun/Software/SFDR/).

\section{Results}

\subsection{SNP Selection}

Step 1: From the 21 loci identified in Lambert et al., (2013) in association with AD, 10 were already known through previous GWAS and 11 novel loci were found (Table 1). APP, PSEN1 and PSEN2 were also added to the gene list. 
Bioinformatics with quantitative phenotype-genotype associations

Step 2: Common biological processes within the gene list were identified using GO. INRICH was used as an alternative objective method, but significant results were not found. Regardless, results from INRICH may be found in the supplementary section. The GO database was accessed on January $27^{\text {th }} 2014$. In the GO database all genes from the list had BP GO terms annotated to them except the gene Membrane-spanning 4-domains subfamily A member 6A (MS4A6A). Table 2 shows the common BP domains associated with the 21 genes. In this study we focused on the "nervous system development and synaptic transmission” network (Figure 4), which included many genes from our original list. The network can be broken down into sub-domains with key GO terms in the areas of synaptic function, neuroanatomical structure development, and neurogenesis. For example, in the domain "neuroanatomical structure development”, myocyte-specific enhancer factor 2C (MEF2C) has been associated with GO terms 'denate gyrus development' and 'nervous system development'.

Step 3: Cytoscape visualization of the nervous system synaptic transmission network is shown in Figure 4.

Step 4: The list of genes associated with the OGO terms from the pruned nervous system and synaptic transmission network included 1249 genes, after removal of all non-autosomal genes 1146 genes remained and formed our stratum for sFDR. Supplementary Table S1 shows a list of all priority genes with chromosome number, start and end position and gene symbol. Furthermore Supplementary Table S2 contains all 249,001 SNPs from 1146 genes used for sFDR. 
Bioinformatics with quantitative phenotype-genotype associations

Table 1. Top genes associated with AD from the Lambert et al., (2013) meta-analysis.

\begin{tabular}{|c|l|}
\hline \multicolumn{2}{|c|}{ Table 1a) 10 loci known to be associated with Alzheimer's disease } \\
\hline Gene Symbol & \multicolumn{1}{c|}{ Gene Name } \\
\hline APOE & Apolipoprotein E \\
\hline BIN1 & Myc box-dependent-interacting protein 1 \\
\hline CLU & Clusterin (Apolipoprotein J) \\
\hline ABCA7 & ATP-binding cassette sub-family A member 7 \\
\hline CR1 & Complement receptor type 1 \\
\hline PICALM & Phosphatidylinositol-binding clathrin assembly protein \\
\hline MS4A6A & Membrane-spanning 4-domains subfamily A member 6A \\
\hline CD33 & Myeloid cell surface antigen CD33 \\
\hline CD2AP & CD2-associated protein \\
\hline EPHA1 & Ephrin type-A receptor 1 \\
\hline \multicolumn{2}{|c|}{ Table 1b) 11 new loci associated Alzheimer's disease } \\
\hline Gene Symbol & \\
\hline HLA & Human leukocyte antigen class II histocompatibility antigen \\
\hline SORL1 & Sortilin-related receptor \\
\hline PTK2B & Protein-tyrosine kinase 2-beta \\
\hline SLC24A4 & Sodium/potassium/calcium exchanger 4 \\
\hline NYAP1 & Neuronal tyrosine-phosphorylated phosphoinositide-3-kinase adapter 1 \\
\hline CELF1 & Encode CUGBP, Elav-like family member 1 region \\
\hline NME8 & Thioredoxin domain-containing protein 3 \\
\hline FERMT2 & Fermitin family homolog 2 \\
\hline INPP5D & Phosphatidylinositol 3,4,5-trisphosphate 5-phosphatase 1 \\
\hline MEF2C & Myocyte-specific enhancer factor 2C \\
\hline CASS4 & Cas scaffolding protein family member 4 \\
\hline
\end{tabular}




\section{Bioinformatics with quantitative phenotype-genotype associations}

Table 2. Common GO Biological Process domains of gene hits from the Lambert et al., (2013) meta-analysis.

\begin{tabular}{|c|c|c|c|c|c|c|c|}
\hline Gene & $\begin{array}{l}\text { Protein } \\
\text { ID }\end{array}$ & $\begin{array}{l}\text { Vesicle- } \\
\text { mediated } \\
\text { transport } \\
\text { and } \\
\text { Endocytosis }\end{array}$ & $\begin{array}{c}\text { Steroid } \\
\text { and } \\
\text { cholesterol } \\
\text { metabolic } \\
\text { process }\end{array}$ & $\begin{array}{c}\text { Immune } \\
\text { system } \\
\text { process }\end{array}$ & $\begin{array}{c}\text { Cell } \\
\text { membrane } \\
\text { processes } \\
\text { and } \\
\text { Cell } \\
\text { migration } \\
\end{array}$ & $\begin{array}{c}\text { Nervous } \\
\text { system } \\
\text { development } \\
\text { and } \\
\text { Synaptic } \\
\text { transmission }\end{array}$ & $\begin{array}{c}\text { Regulation } \\
\text { of } \\
\text { calcium- } \\
\text { mediated } \\
\text { signaling }\end{array}$ \\
\hline DRB5 & Q30154 & & & $X$ & & & \\
\hline SORL1 & Q92673 & $X$ & $X$ & & $X$ & & \\
\hline PTK2B & Q14289 & & & $X$ & $X$ & $X$ & $X$ \\
\hline SLC24A4 & Q8NFF2 & $X$ & & & & & $X$ \\
\hline NYAP1 & Q6ZVC0 & & & & & $\mathrm{X}$ & \\
\hline MADD & Q8WXG6 & & & & $X$ & & \\
\hline NME8 & Q8N427 & & & & $X$ & & \\
\hline FERMT2 & Q96AC1 & $X$ & & & $X$ & & \\
\hline INPP5D & Q92835 & & & $X$ & & & \\
\hline MEF2C & Q06413 & & & & & $X$ & \\
\hline CASS4 & Q9NQ75 & & & & & & \\
\hline APOE & P02649 & $X$ & $X$ & & $X$ & $X$ & $X$ \\
\hline BIN1 & O00499 & $X$ & & & $X$ & & \\
\hline CLU & P10909 & $X$ & $X$ & $X$ & & & \\
\hline ABCA7 & Q8IZY2 & $X$ & $X$ & $X$ & & & \\
\hline CR1 & P17927 & & & $X$ & & & \\
\hline PICALM & Q13492 & $X$ & & $X$ & & $X$ & \\
\hline MS4A6A & Q9H2W1 & & & & & & \\
\hline CD33 & P20138 & & & $X$ & $\mathrm{X}$ & & \\
\hline CD2AP & Q9Y5K6 & & & & $\mathrm{X}$ & & \\
\hline EPHA1 & P21709 & & & $X$ & $X$ & & \\
\hline
\end{tabular}

\subsection{Quality Control of Imaging and GWAS data}

After quality control (QC) of automatic hippocampal segmentations, 9 segmentations out of 662 subjects failed which were corrected though manual segmentation. For the ADNI1 GWAS data, the sample initially consisted of 757 individuals, and after QC the sample was reduced to 662 subjects. The number of SNPs in the GWAS data after QC was 529,623 from 620,901 original variants, of which 517,064 SNPs were on autosomal chromosomes. After imputation of the GWAS, data the number of SNPs typed increased to 17,418,272. After QC of imputed SNPs, 5,706,558 SNPs were used for the association analysis with mean hippocampal volume.

\subsection{Association Testing with Hippocampal Volume}

P-values from association testing between the SNPs and mean hippocampal volume did not result in any GWAS significant findings after correction for multiple testing (Figure 5). Some, however, approached significance (Table 3, SNPs with uncorrected p-values). For example rs72909661 in gene region Stearoyl-CoA desaturase 5 (SCD5) neared GWAS significance with an uncorrected $\mathrm{p}=$ 
Bioinformatics with quantitative phenotype-genotype associations

$8.97 \times 10^{-7}$. The top 10 SNPs found within gene regions were: Autism susceptibility gene 2 protein (AUTS2; rs2158616; $\mathrm{p}=1.16 \times 10^{-6}$ ), Transmembrane protein - family with sequence similarity 155 member A (FAM155A; rs1033880; $\mathrm{p}=4.42 \times 10^{-6}$ ) and long non-coding RNAs (LOC440173; rs11791915; $\mathrm{p}=1.76 \times 10^{-6}$ ). Testing with left and right hippocampal volumes as response variables resulted in no GWAS significant findings, as displayed in the supplemental materials.

\section{Table 3. Top 10 most significant SNPs within gene regions from association testing of mean} hippocampal volume

MAF represents minor allele frequency and p-value is the associated significance between the SNP and phenotype (mean hippocampal volume). Significant SNPs at a GWAS level are at $\mathrm{p}<5 \times 10^{-8}$.

\begin{tabular}{|c|c|c|c|c|c|c|}
\hline $\begin{array}{c}\text { Chromosome } \\
\text { Number }\end{array}$ & SNP ID & $\begin{array}{c}\text { Base } \\
\text { position }\end{array}$ & MAF & p-value & $\begin{array}{c}\text { NCBI Gene } \\
\text { ID }\end{array}$ & Gene Symbol \\
\hline 9 & rs75592689 & 89700141 & 0.07 & $6.92 E-07$ & 494127 & LOC494127 \\
\hline 4 & rs72909661 & 83680826 & 0.05 & $8.97 E-07$ & 79966 & SCD5 \\
\hline 7 & rs2158616 & 70075454 & 0.10 & $1.16 E-06$ & 26053 & AUTS2 \\
\hline 7 & rs2158617 & 70075447 & 0.10 & $1.23 E-06$ & 26053 & AUTS2 \\
\hline 7 & rs2158618 & 70075402 & 0.10 & $1.60 E-06$ & 26053 & AUTS2 \\
\hline 9 & rs11791915 & 89643751 & 0.08 & $1.76 E-06$ & 440173 & LOC440173 \\
\hline 9 & rs11789129 & 89638584 & 0.07 & $2.42 E-06$ & 440173 & LOC440173 \\
\hline 13 & rs1033880 & 108222156 & 0.44 & $4.42 E-06$ & 728215 & FAM155A \\
\hline 13 & rs9520494 & 108222992 & 0.44 & $6.00 E-06$ & 728215 & FAM155A \\
\hline 13 & rs9520495 & 108224804 & 0.44 & $6.79 E-06$ & 728215 & FAM155A \\
\hline
\end{tabular}

\section{4 sFDR Results}

In total there were 249,001 SNPs in our priority stratum and 5,457,557 SNPs in our non-priority stratum. No q-values from the priority list (nervous system development and synaptic transmission stratum) reached the 0.05 threshold. All of the top 97 ranked SNPs were found in our priority list, but these SNPs were not significant at a q-value of less than 0.05. In particular, SNPs in our priority list within gene regions: $\mathrm{C} 2$ calcium-dependent domain containing 3 (CDCD3), growth arrest-specific (GAS7), Semaphorin-3D (SEMA3D), Gap junction alpha-1 protein (GJA1) and Rap guanine nucleotide exchange factor 1 (RAPGEF1) were ranked in the top 10 but with an sFDR q-value of 0.71 (Table 4). Both the priority and non-priority stratum display uniform p-values; this indicates that there is no true association between the SNPs and mean hippocampal volume (Supplementary Figure S1 and S2). 


\section{Bioinformatics with quantitative phenotype-genotype associations}

349

350

351

352

353

\section{Table 4. Top 10 most significant sFDR results for mean hippocampal volume}

P-value is the associated significance between the SNP and phenotype (mean hippocampal volume). Significant SNPs at a GWAS level is $\mathrm{p}<5 \times 10^{-8}$. The sFDR q-value controls the false discovery rate; the q-value is the adjusted p-value. Significant q-value is set to 0.05. 'Rank' is the order of SNPs based on sFDR q-values from a total of 5,706,558 SNPs.

\begin{tabular}{|c|c|c|c|c|c|c|c|}
\hline $\begin{array}{c}\text { Chromosome } \\
\text { number }\end{array}$ & SNP ID & $\begin{array}{c}\text { Base } \\
\text { position }\end{array}$ & p-value & q-value & rank & $\begin{array}{c}\text { NCBI } \\
\text { Gene ID }\end{array}$ & $\begin{array}{c}\text { Gene } \\
\text { Symbol }\end{array}$ \\
\hline 11 & rs12417424 & 73751481 & $2.94 \mathrm{E}-05$ & 0.7113 & 1 & 26005 & C2CD3 \\
\hline 17 & rs62064532 & 9850440 & $3.17 \mathrm{E}-05$ & 0.7113 & 2 & 8522 & GAS7 \\
\hline 11 & rs4944877 & 73753671 & $3.74 \mathrm{E}-05$ & 0.7113 & 3 & 26005 & C2CD3 \\
\hline 11 & rs58006161 & 73860874 & $4.05 E-05$ & 0.7113 & 4 & 26005 & C2CD3 \\
\hline 11 & rs58719576 & 73860873 & $4.05 E-05$ & 0.7113 & 5 & 26005 & C2CD3 \\
\hline 7 & rs17558985 & 84643942 & $4.19 E-05$ & 0.7113 & 6 & 223117 & SEMA3D \\
\hline 17 & rs55753206 & 9856314 & $6.34 \mathrm{E}-05$ & 0.7113 & 7 & 8522 & GAS7 \\
\hline 6 & rs113413235 & 121762030 & $6.35 E-05$ & 0.7113 & 8 & 2697 & GJA1 \\
\hline 9 & rs7469510 & 134559741 & $6.37 E-05$ & 0.7113 & 9 & 2889 & RAPGEF1 \\
\hline
\end{tabular}

354

\section{Discussion}

In contrast to existing approaches, our novel method provides a systematic integration framework for previous knowledge with the GO database. Alternatives such as Aligator (Holmans et al., 2009) and INRICH both rely on the identification of over-represented GO categories among significant hits; we identify and adapt relevant categories and use sFDR to increase power while controlling for multiplicity.

With no individual variants reaching genome-wide significance, we suspect a lack of statistical power even while employing the sFDR framework. In the future, our method will be adapted and applied to different datasets, and specifically, alternate phenotypes of AD. The high concentration of amyloid plaques in subcortical structures in late stage AD and neurofibrillary tangles originating in the medial temporal lobe implicate these regions as potential targets for future analysis (Braak and Braak, 1991).

The process by which priority SNPs are selected for sFDR could be considered subjective and represents an area of active development for our algorithm. Often the associations in GWAS studies are designated to the most promising gene in the region from a biological standpoint, introducing bias in step 1. One approach to combat this phenomenon would be for the input list to include all genes within a high recombination region alongside the most significant hit. Selection of the common biological domains in step 2 is also subjective and could be replaced with a standard pathway approach such as INRICH or Aligator. We have performed pilot work using INRICH as outlined in the supplementary text. In this example no pathways were identified, preventing us from pursuing this avenue. This is likely due to a lack of power to identify relevant pathways.

Another area of active development relating to SNP selection revolves around growing and pruning the network of terms utilized. Specifically, the GO terms in the "nervous system development and synaptic transmission” domain are general, and apply towards whole brain structure; currently the ontology does not capture particular brain regions in details such as hippocampal development. For example, in our priority list, SNPs in gene region growth arrest-specific protein 7 which plays a role 
in neuronal development are mainly expressed in mature cerebellar Purkinje cells (Ju et al., 1998). As such, further pruning the GO network by focusing on BP GO terms annotated to genes specific to one brain region, such as the hippocampus, or neuronal cell type within such structures may be crucial. Biological processes associated with structural information have recently begun to be captured in GO (Huntley et al., 2014). Therefore, when genes are annotated to BP GO terms, additional information on where the biological process is occurring can be recorded. As a result, filtering the data and looking at BP GO terms occurring in neuro-anatomical cells in region of the hippocampus may help in further pruning the network.

Both automatic and manual curation was used to assign GO terms to the genes in question. Automatic curation is the result of machine learning algorithms, and the terms assigned tend to be much broader than the manually curated ones and adds a potential source of noise to our priority SNPs stratum. In the example analysis presented here the inclusion of these sub-optimal classifications was necessary due to the limited manual annotation of the loci observed in Lambert et al., yet we acknowledge the shortcomings of this approach and advise the prioritization of manually curated data GO data. To further address the issue we are in the process of manually curating the list of 21 loci associated with AD.

In conclusion, this article introduces the use of GO, an online database, as a novel method to efficiently prioritize data for sFDR multiple testing control. In particular we applied this method to a GWAS of hippocampal volume in the ADNI1 dataset. Though novel biomarkers were not identified, our method has the potential to improve the identification of genes in imaging-genetic studies.

\section{Conflict of Interest}

We declare that the research was conducted in the absence of any commercial or financial relationships that could be construed as a potential conflict of interest.

\section{Acknowledgments}

Computations were performed on the CAMH Specialized Computing Cluster. The SCC is funded by: The Canada Foundation for Innovation, Research Hospital Fund. Computations were performed on the gpc supercomputer at the SciNet HPC Consortium (Loken et al., 2010). SciNet is funded by: the Canada Foundation for Innovation under the auspices of Compute Canada; the Government of Ontario; Ontario Research Fund - Research Excellence; and the University of Toronto. MMC is funded by the National Sciences and Engineering Research Council, Canadian Institutes for Health, Weston Brain Institute, Michael J. Fox Foundation for Parkinson’s Research, Alzheimer's Society, and Brain Canada. JK holds the Joanne Murphy Professorship in Behavioral Science and is also funded by the Canadian Institutes for Health. Sejal Patel is funded by The Cadsby Foundation. Thanks to Mark Silverberg for providing the Jewish ancestry sample. We would like to thank Chris Coles for his help with editing the manuscript.

ADNI Acknowledgments: Data collection and sharing for this project was funded by the Alzheimer's Disease Neuroimaging Initiative (ADNI) (National Institutes of Health Grant U01 AG024904) and DOD ADNI (Department of Defense award number W81XWH-12-2-0012). ADNI is funded by the National Institute on Aging, the National Institute of Biomedical Imaging and Bioengineering, and through generous contributions from the following: Alzheimer's Association; Alzheimer's Drug Discovery Foundation; BioClinica, Inc.; Biogen Idec Inc.; Bristol-Myers Squibb Company; Eisai Inc.; Elan Pharmaceuticals, Inc.; Eli Lilly and Company; F. Hoffmann-La Roche Ltd. and its 
Bioinformatics with quantitative phenotype-genotype associations

429

430

431

432

433

434

435

436

437

438

439

440

441

442

443

444

445

446

447

448

449

450

451

452

453

454

455

456

457

458

459

460

461

462

463

464

465

466

467

468

469

470

471

472

473

affiliated company Genentech, Inc.; GE Healthcare; Innogenetics, N.V.; IXICO Ltd.; Janssen Alzheimer Immunotherapy Research \& Development, LLC.; Johnson \& Johnson Pharmaceutical Research \& Development LLC.; Medpace, Inc.; Merck \& Co., Inc.; Meso Scale Diagnostics, LLC.; NeuroRx Research; Novartis Pharmaceuticals Corporation; Pfizer Inc.; Piramal Imaging; Servier; Synarc Inc.; and Takeda Pharmaceutical Company. The Canadian Institutes of Health Research is providing funds to support ADNI clinical sites in Canada. Private sector contributions are facilitated by the Foundation for the National Institutes of Health (www.fnih.org). The grantee organization is the Northern California Institute for Research and Education, and the study is coordinated by the Alzheimer's Disease Cooperative Study at the University of California, San Diego. ADNI data are disseminated by the Laboratory for Neuro Imaging at the University of Southern California.

\section{Author Contributions}

SP performed quality control and imputation on GWAS data, developed the Gene Ontology network and performed the association analysis between GWAS data and mean hippocampal volume. MTMP carried out automatic and manual hippocampal segmentation and quality control on the segmentation. SP, JK, MC conceptualized the design of the study. All authors wrote and edited the manuscript.

\section{References}

Altshuler, D.M., Gibbs, R.A., Peltonen, L., Dermitzakis, E., Schaffner, S.F., Yu, F., et al. (2010). Integrating common and rare genetic variation in diverse human populations. Nature 467, 5258. doi: 10.1038/nature09298.

Ashburner, M., Ball, C.A., Blake, J.A., Botstein, D., Butler, H., Cherry, J.M., et al. (2000). Gene ontology: tool for the unification of biology. The Gene Ontology Consortium. Nature genetics 25, 25-29. doi: 10.1038/75556.

Avants, B.B., Epstein, C.L., Grossman, M., and Gee, J.C. (2008). Symmetric diffeomorphic image registration with cross-correlation: evaluating automated labeling of elderly and neurodegenerative brain. Medical image analysis 12, 26-41. doi: 10.1016/j.media.2007.06.004.

Benjamini, Y., and Hochberg, Y. (1995). Controlling the false discovery rate: a practical and powerful approach to multiple testing. Journal of the Royal Statistical Society. Series B (Methodological), 289-300.

Braak, H., and Braak, E. (1991). Neuropathological stageing of Alzheimer-related changes. Acta neuropathologica 82, 239-259.

Braskie, M.N., Toga, A.W., and Thompson, P.M. (2013). Recent advances in imaging Alzheimer's disease. Journal of Alzheimer's disease : JAD 33 Suppl 1, S313-327. doi: 10.3233/JAD-2012129016.

Chakravarty, M.M., Steadman, P., Van Eede, M.C., Calcott, R.D., Gu, V., Shaw, P., et al. (2013). Performing label-fusion-based segmentation using multiple automatically generated templates. Human brain mapping 34, 2635-2654. doi: 10.1002/hbm.22092.

Collins, D.L., and Pruessner, J.C. (2010). Towards accurate, automatic segmentation of the hippocampus and amygdala from MRI by augmenting ANIMAL with a template library and label fusion. NeuroImage 52, 1355-1366. doi: 10.1016/j.neuroimage.2010.04.193.

Cruchaga, C., Haller, G., Chakraverty, S., Mayo, K., Vallania, F.L., Mitra, R.D., et al. (2012). Rare variants in APP, PSEN1 and PSEN2 increase risk for AD in late-onset Alzheimer's disease families. PloS one 7, e31039. doi: 10.1371/journal.pone.0031039. 
Cruts, M., Van Duijn, C.M., Backhovens, H., Van Den Broeck, M., Wehnert, A., Serneels, S., et al. (1998). Estimation of the genetic contribution of presenilin-1 and -2 mutations in a population-based study of presenile Alzheimer disease. Human molecular genetics 7, 43-51.

Delaneau, O., Marchini, J., and Zagury, J.F. (2012). A linear complexity phasing method for thousands of genomes. Nature methods 9, 179-181. doi: 10.1038/nmeth.1785.

Dudbridge, F., and Gusnanto, A. (2008). Estimation of significance thresholds for genomewide association scans. Genetic epidemiology 32, 227-234. doi: 10.1002/gepi.20297.

Farrer, L.A., Cupples, L.A., Haines, J.L., Hyman, B., Kukull, W.A., Mayeux, R., et al. (1997). Effects of age, sex, and ethnicity on the association between apolipoprotein E genotype and Alzheimer disease. A meta-analysis. APOE and Alzheimer Disease Meta Analysis Consortium. JAMA 278, 1349-1356.

Goate, A., Chartier-Harlin, M.C., Mullan, M., Brown, J., Crawford, F., Fidani, L., et al. (1991). Segregation of a missense mutation in the amyloid precursor protein gene with familial Alzheimer's disease. Nature 349, 704-706. doi: 10.1038/349704a0.

Heckemann, R.A., Hajnal, J.V., Aljabar, P., Rueckert, D., and Hammers, A. (2006). Automatic anatomical brain MRI segmentation combining label propagation and decision fusion. NeuroImage 33, 115-126. doi: 10.1016/j.neuroimage.2006.05.061.

Hirschhorn, J.N., Lohmueller, K., Byrne, E., and Hirschhorn, K. (2002). A comprehensive review of genetic association studies. Genetics in medicine : official journal of the American College of Medical Genetics 4, 45-61. doi: 10.109700125817-200203000-00002.

Holmans, P., Green, E.K., Pahwa, J.S., Ferreira, M.A., Purcell, S.M., Sklar, P., et al. (2009). Gene ontology analysis of GWA study data sets provides insights into the biology of bipolar disorder. American journal of human genetics 85, 13-24. doi: 10.1016/j.ajhg.2009.05.011.

Huntley, R.P., Harris, M.A., Alam-Faruque, Y., Blake, J.A., Carbon, S., Dietze, H., et al. (2014). A method for increasing expressivity of Gene Ontology annotations using a compositional approach. BMC bioinformatics 15, 155. doi: 10.1186/1471-2105-15-155.

Ioannidis, J.P. (2005). Why most published research findings are false. PLoS medicine 2, e124. doi: 10.1371/journal.pmed.0020124.

Jack, C.R., Jr., Bernstein, M.A., Fox, N.C., Thompson, P., Alexander, G., Harvey, D., et al. (2008). The Alzheimer's Disease Neuroimaging Initiative (ADNI): MRI methods. Journal of magnetic resonance imaging : JMRI 27, 685-691. doi: 10.1002/jmri.21049.

Ju, Y.T., Chang, A.C., She, B.R., Tsaur, M.L., Hwang, H.M., Chao, C.C., et al. (1998). gas7: A gene expressed preferentially in growth-arrested fibroblasts and terminally differentiated Purkinje neurons affects neurite formation. Proceedings of the National Academy of Sciences of the United States of America 95, 11423-11428.

Lambert, J.C., Ibrahim-Verbaas, C.A., Harold, D., Naj, A.C., Sims, R., Bellenguez, C., et al. (2013). Meta-analysis of 74,046 individuals identifies 11 new susceptibility loci for Alzheimer's disease. Nature genetics 45, 1452-1458. doi: 10.1038/ng.2802.

Lander, E.S., Linton, L.M., Birren, B., Nusbaum, C., Zody, M.C., Baldwin, J., et al. (2001). Initial sequencing and analysis of the human genome. Nature 409, 860-921. doi: 10.1038/35057062.

Lee, P.H., O'dushlaine, C., Thomas, B., and Purcell, S.M. (2012). INRICH: interval-based enrichment analysis for genome-wide association studies. Bioinformatics 28, 1797-1799. doi: 10.1093/bioinformatics/bts191.

Loken, C., Gruner, D., Groer, L., Peltier, R., Bunn, N., Craig, M., et al. (Year). "SciNet: lessons learned from building a power-efficient top-20 system and data centre", in: Journal of Physics: Conference Series: IOP Publishing), 012026. 
Bioinformatics with quantitative phenotype-genotype associations

Marchini, J., Howie, B., Myers, S., Mcvean, G., and Donnelly, P. (2007). A new multipoint method for genome-wide association studies by imputation of genotypes. Nature genetics 39, 906913. doi: 10.1038/ng2088.

Patel, V., Chiang, M.-C., Thompson, P.M., Mcmahon, K.L., De Zubicaray, G.I., Martin, N.G., et al. (Year). "Scalar connectivity measures from fast-marching tractography reveal heritability of white matter architecture", in: Biomedical Imaging: From Nano to Macro, 2010 IEEE International Symposium on: IEEE), 1109-1112.

Pipitone, J., Park, M.T., Winterburn, J., Lett, T.A., Lerch, J.P., Pruessner, J.C., et al. (2014). Multiatlas segmentation of the whole hippocampus and subfields using multiple automatically generated templates. NeuroImage 101, 494-512. doi: 10.1016/j.neuroimage.2014.04.054.

Purcell, S., Neale, B., Todd-Brown, K., Thomas, L., Ferreira, M.A., Bender, D., et al. (2007). PLINK: a tool set for whole-genome association and population-based linkage analyses. American journal of human genetics 81, 559-575. doi: 10.1086/519795.

Saito, R., Smoot, M.E., Ono, K., Ruscheinski, J., Wang, P.L., Lotia, S., et al. (2012). A travel guide to Cytoscape plugins. Nature methods 9, 1069-1076. doi: 10.1038/nmeth.2212.

Stein, J.L., Medland, S.E., Vasquez, A.A., Hibar, D.P., Senstad, R.E., Winkler, A.M., et al. (2012). Identification of common variants associated with human hippocampal and intracranial volumes. Nature genetics 44, 552-561. doi: 10.1038/ng.2250.

Sun, L., Craiu, R.V., Paterson, A.D., and Bull, S.B. (2006). Stratified false discovery control for large-scale hypothesis testing with application to genome-wide association studies. Genetic epidemiology 30, 519-530. doi: 10.1002/gepi.20164.

Sun, L., Rommens, J.M., Corvol, H., Li, W., Li, X., Chiang, T.A., et al. (2012). Multiple apical plasma membrane constituents are associated with susceptibility to meconium ileus in individuals with cystic fibrosis. Nature genetics 44, 562-569. doi: 10.1038/ng.2221.

Tabor, H.K., Risch, N.J., and Myers, R.M. (2002). Candidate-gene approaches for studying complex genetic traits: practical considerations. Nature reviews. Genetics 3, 391-397. doi: 10.1038/nrg796.

Thompson, P.M., Martin, N.G., and Wright, M.J. (2010). Imaging genomics. Current opinion in neurology 23, 368-373. doi: 10.1097/WCO.0b013e32833b764c.

Wellcome_Trust_Case_Control_Consortium (2007). Genome-wide association study of 14,000 cases of seven common diseases and 3,000 shared controls. Nature 447, 661-678. doi: 10.1038/nature05911.

Winterburn, J.L., Pruessner, J.C., Chavez, S., Schira, M.M., Lobaugh, N.J., Voineskos, A.N., et al. (2013). A novel in vivo atlas of human hippocampal subfields using high-resolution $3 \mathrm{~T}$ magnetic resonance imaging. NeuroImage 74, 254-265. doi: 10.1016/j.neuroimage.2013.02.003.

Wyman, B.T., Harvey, D.J., Crawford, K., Bernstein, M.A., Carmichael, O., Cole, P.E., et al. (2013). Standardization of analysis sets for reporting results from ADNI MRI data. Alzheimer's \& dementia : the journal of the Alzheimer's Association 9, 332-337. doi: 10.1016/j.jalz.2012.06.004. 
bioRxiv preprint doi: https://doi.org/10.1101/015065; this version posted February 10, 2015. The copyright holder for this preprint (which was not certified by peer review) is the author/funder, who has granted bioRxiv a license to display the preprint in perpetuity. It is made available under aCC-BY-NC-ND 4.0 International license.

Bioinformatics with quantitative phenotype-genotype associations

Figure Legends

561 Figure 1. Method overview of both the selection of priority SNPs and association testing analysis between ADNI1 GWAS and imaging data. (A) Steps taken to select for priority SNPs. Gene hits from a meta-analysis by Lambert et al., (2013) were used as a starting point (Step 1) and GO was then used to identify common biological processes within the gene hits (Step 2). Cytoscape was used to build and visualize common biological process networks -- in this case the "nervous development and synaptic transmission” network was selected (Step 3 and Step 4). All genes from the selected GO terms in the network were extracted to form the priority list of SNPs. sFDR was then implemented with the priority SNPs. (B) Shows quality controls steps taken GWAS data and hippocampal imaging data. Association analysis was performed between imputed quality control (QC) GWAS data with

570 QCed hippocampal segmentation.

\section{A}

Step 1: Lambert et al (2013) GWAS meta-analysis: Identify top gene hits associated with $A D$

Step 2: Gene Ontology (GO): To find common biological processes within the gene list

571

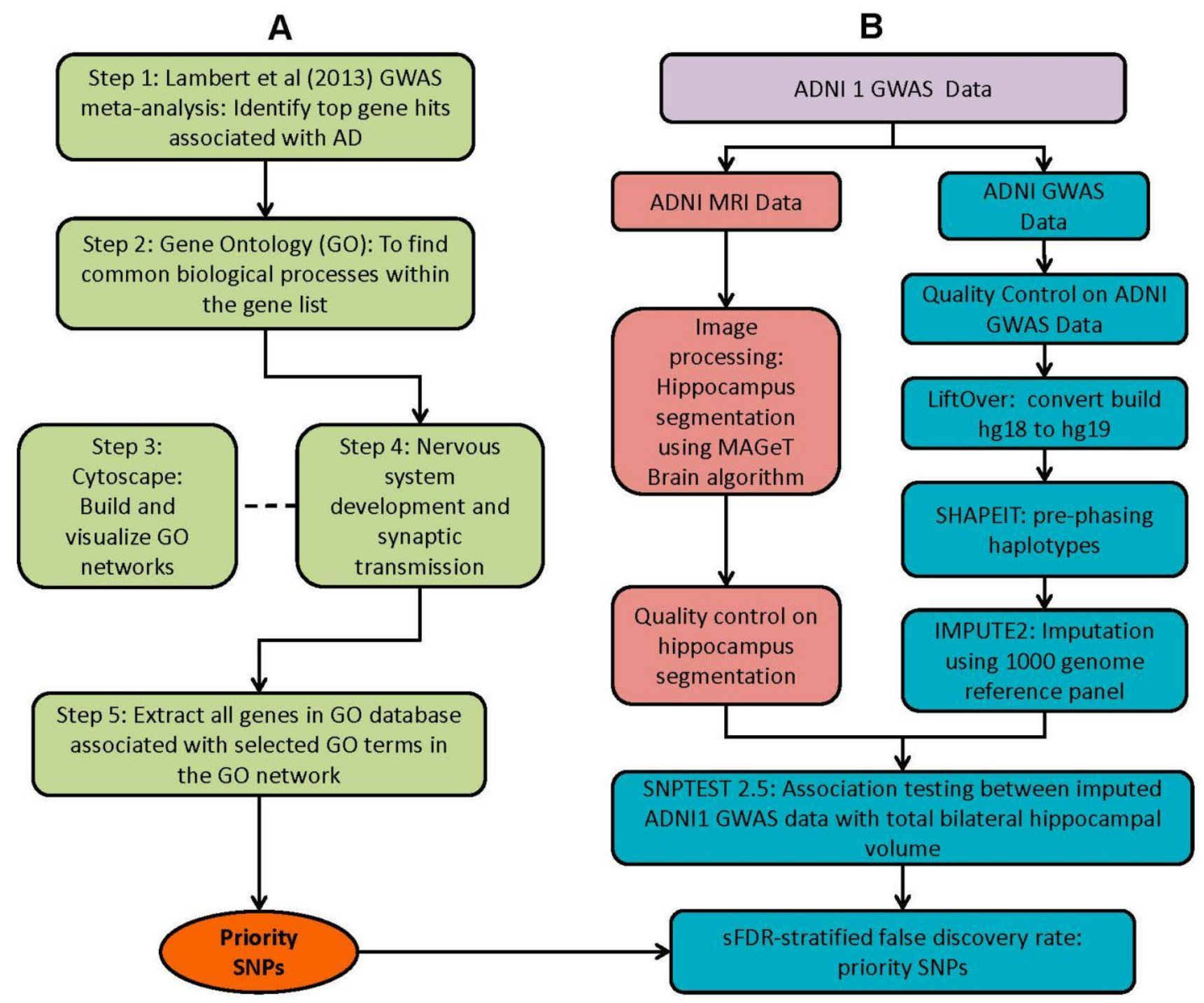


bioRxiv preprint doi: https://doi.org/10.1101/015065; this version posted February 10, 2015. The copyright holder for this preprint (which was not certified by peer review) is the author/funder, who has granted bioRxiv a license to display the preprint in perpetuity. It is made available under aCC-BY-NC-ND 4.0 International license.

\section{Bioinformatics with quantitative phenotype-genotype associations}

572 Figure 2. Sample of initial network with selected GO terms before pruning. A subsection is selected to show how the criteria was used to prune the complex GO network. Pruning steps are shown in

574 Figure 3.

.

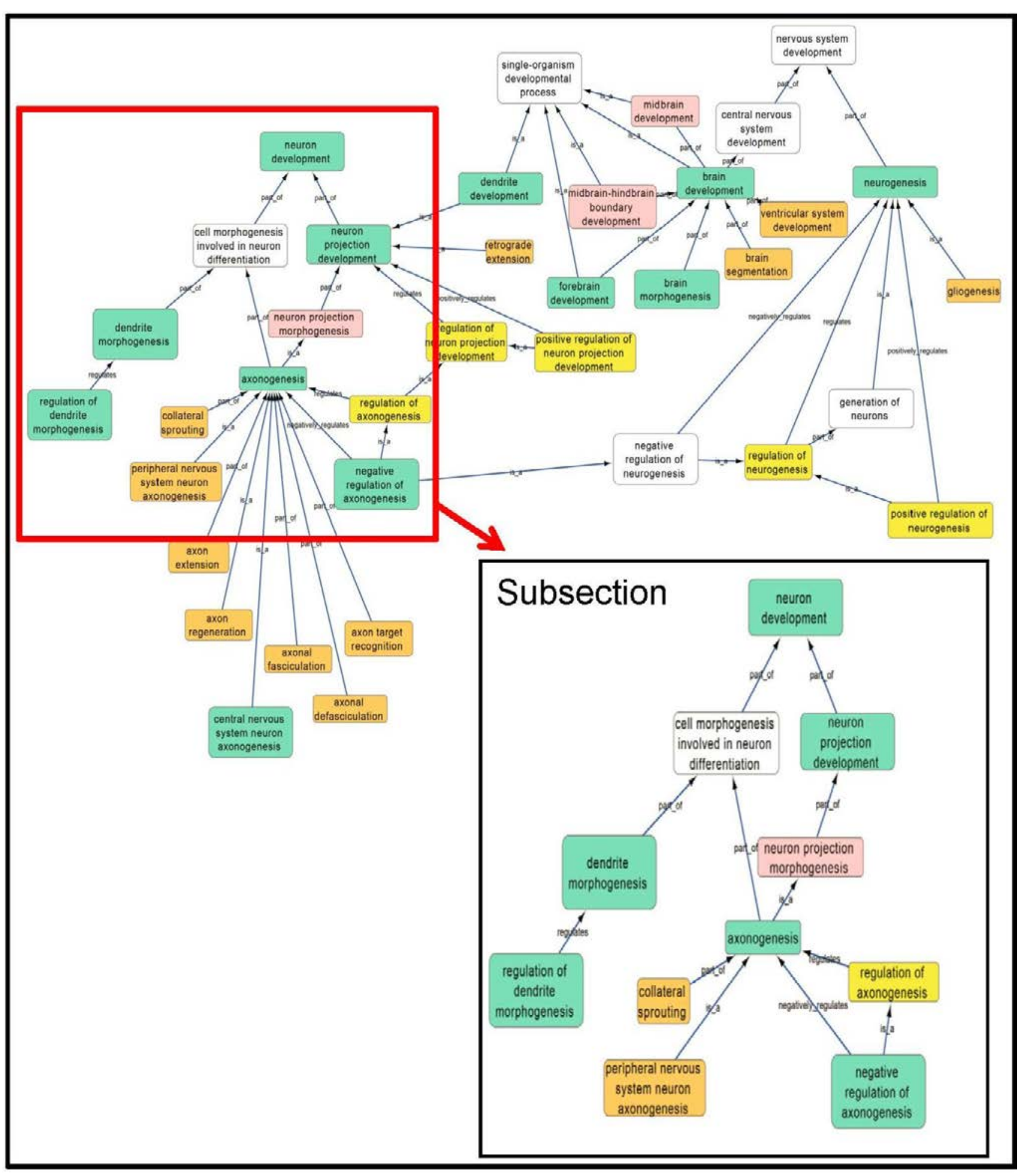


bioRxiv preprint doi: https://doi.org/10.1101/015065; this version posted February 10, 2015. The copyright holder for this preprint (which was not certified by peer review) is the author/funder, who has granted bioRxiv a license to display the preprint in perpetuity. It is made available under aCC-BY-NC-ND 4.0 International license.

Bioinformatics with quantitative phenotype-genotype associations

577 Figure 3. Criteria used to prune a complex network. Green box: Selected GO terms that are associated with a gene identified from Lambert et al., (2013). Orange box: Child terms of selected GO terms. Pink box: Less common parent term only associated with one selected parent GO term. Yellow box: Regulation GO terms that do not specify positive or negative regulation. (A) Child terms of selected GO terms were removed. (B) A less common parent GO term (neuron projection morphogenesis) which has one selected child GO term ('axongenesis') is removed because 'Cell morphogenesis involved in neuron differentiation' is a parent term for both selected GO terms 'axonogenesis' and 'dendrite morphogenesis'. (C) Regulation terms that does not specify the type of regulation is removed because selected GO term 'negative regulation of axonogensis" is more descriptive than the parent GO term 'regulation of axongenesis'. (D) A sample of a pruned network after following the criteria in Figure 3A -3C.

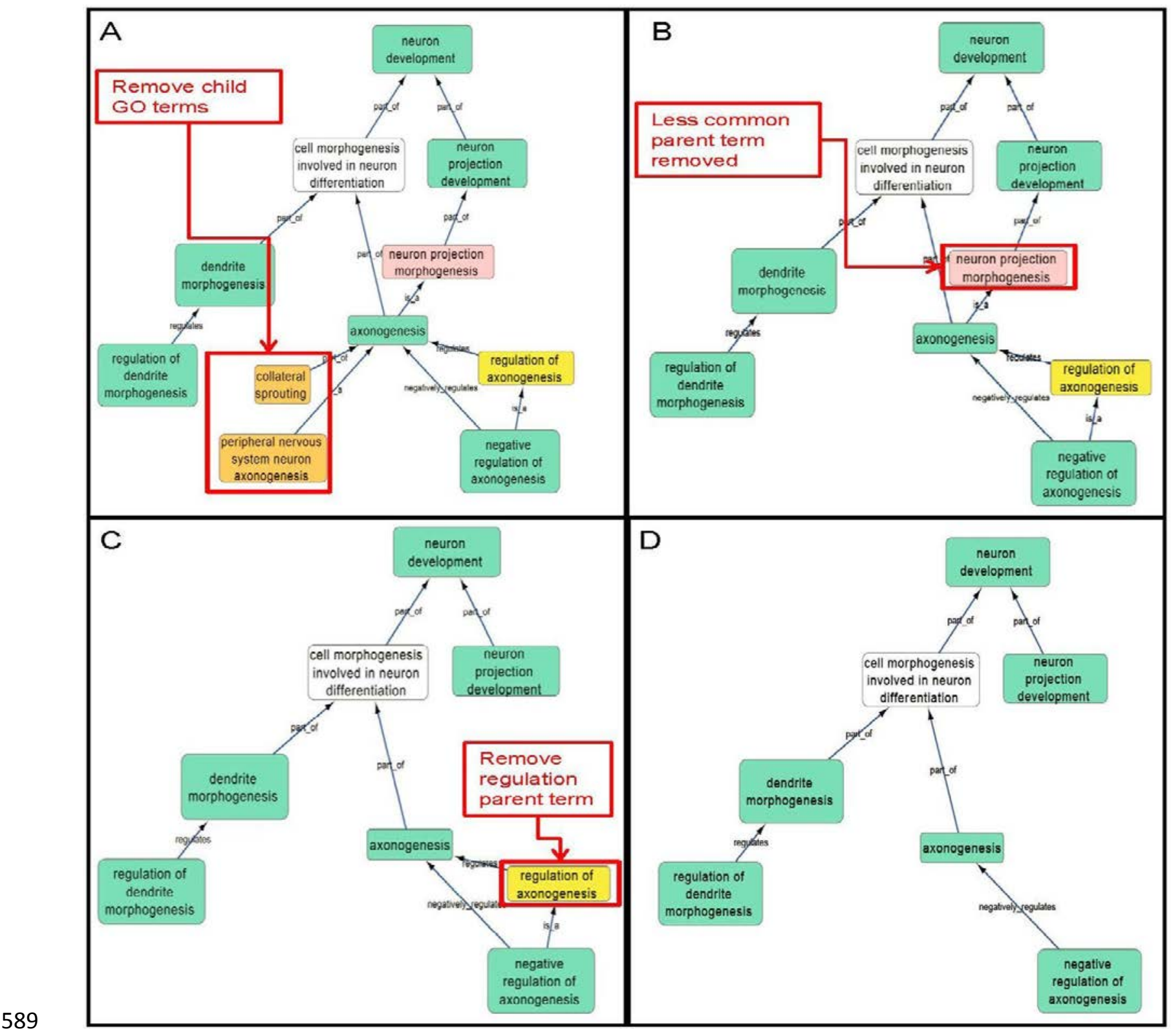


bioRxiv preprint doi: https://doi.org/10.1101/015065; this version posted February 10, 2015. The copyright holder for this preprint (which was not certified by peer review) is the author/funder, who has granted bioRxiv a license to display the preprint in perpetuity. It is made available under aCC-BY-NC-ND 4.0 International license.

\section{Bioinformatics with quantitative phenotype-genotype associations}

Figure 4. Gene Ontology (GO) biological process network of the "nervous system development and synaptic transmission" in association with AD. Green boxes are GO terms that are associated with the specific genes (blue ovals) connected by purple dotted line. White boxes are intermediate parent GO terms related to the selected GO terms (green boxes). Black arrows represent 'is_a' relationship between the GO terms and its parent term; blue arrows shows a 'part _of' relationship; orange arrows, a 'regulation' relationship; green arrows, a 'positive_regulation' relationship and red arrows, a negative_regulation' relationship.

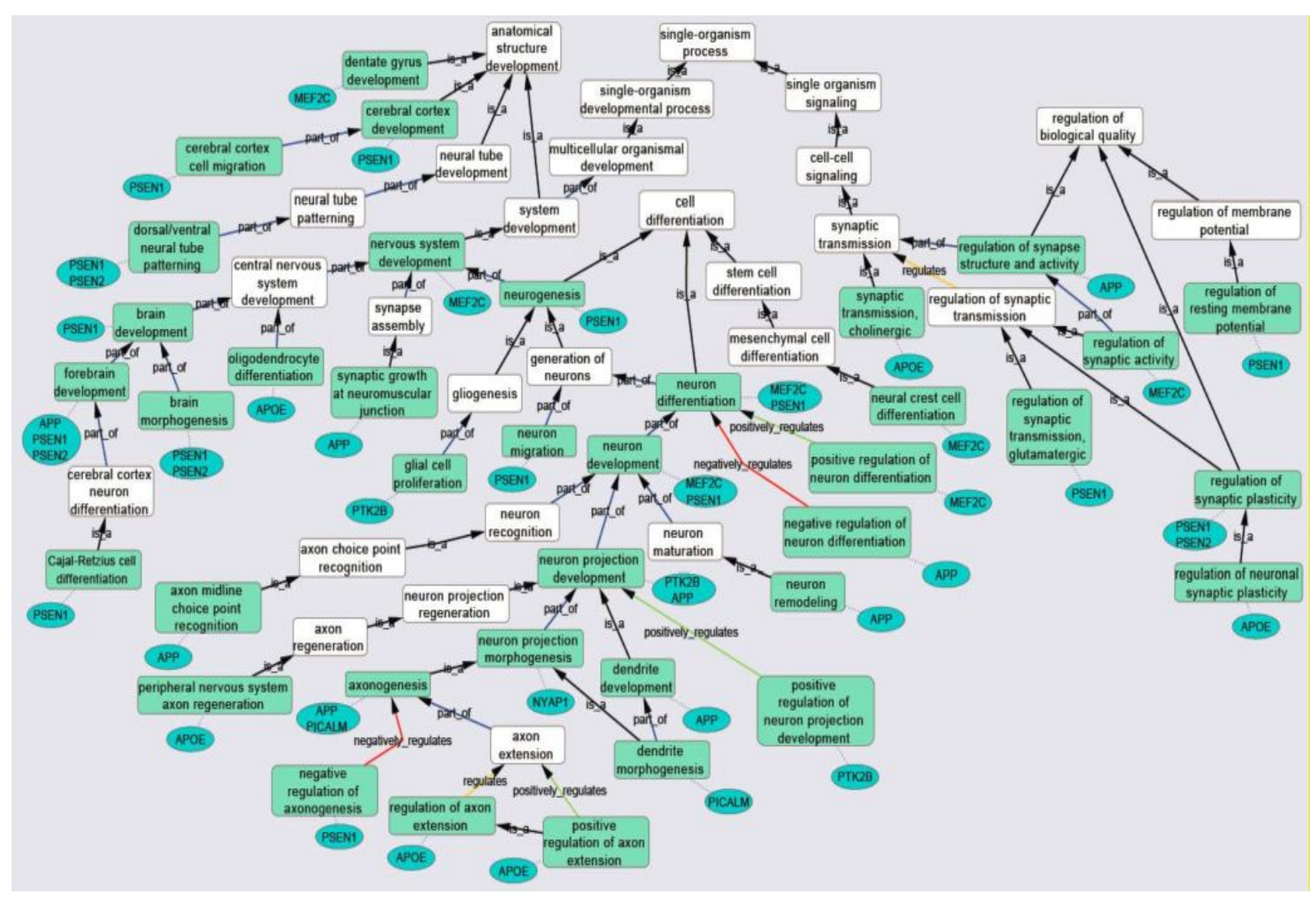


bioRxiv preprint doi: https://doi.org/10.1101/015065; this version posted February 10, 2015. The copyright holder for this preprint (which was not certified by peer review) is the author/funder, who has granted bioRxiv a license to display the preprint in perpetuity. It is made available under aCC-BY-NC-ND 4.0 International license.

Bioinformatics with quantitative phenotype-genotype associations

602

603

604

605

Figure 5. A Manhattan plot of imputed ADNI1 GWAS data. The x-axis represents the chromosomal location for each SNPs. The y-axis represents the log p-values of SNPs in association with AD. The red horizontal line represents the threshold for GWAS significant SNPs.

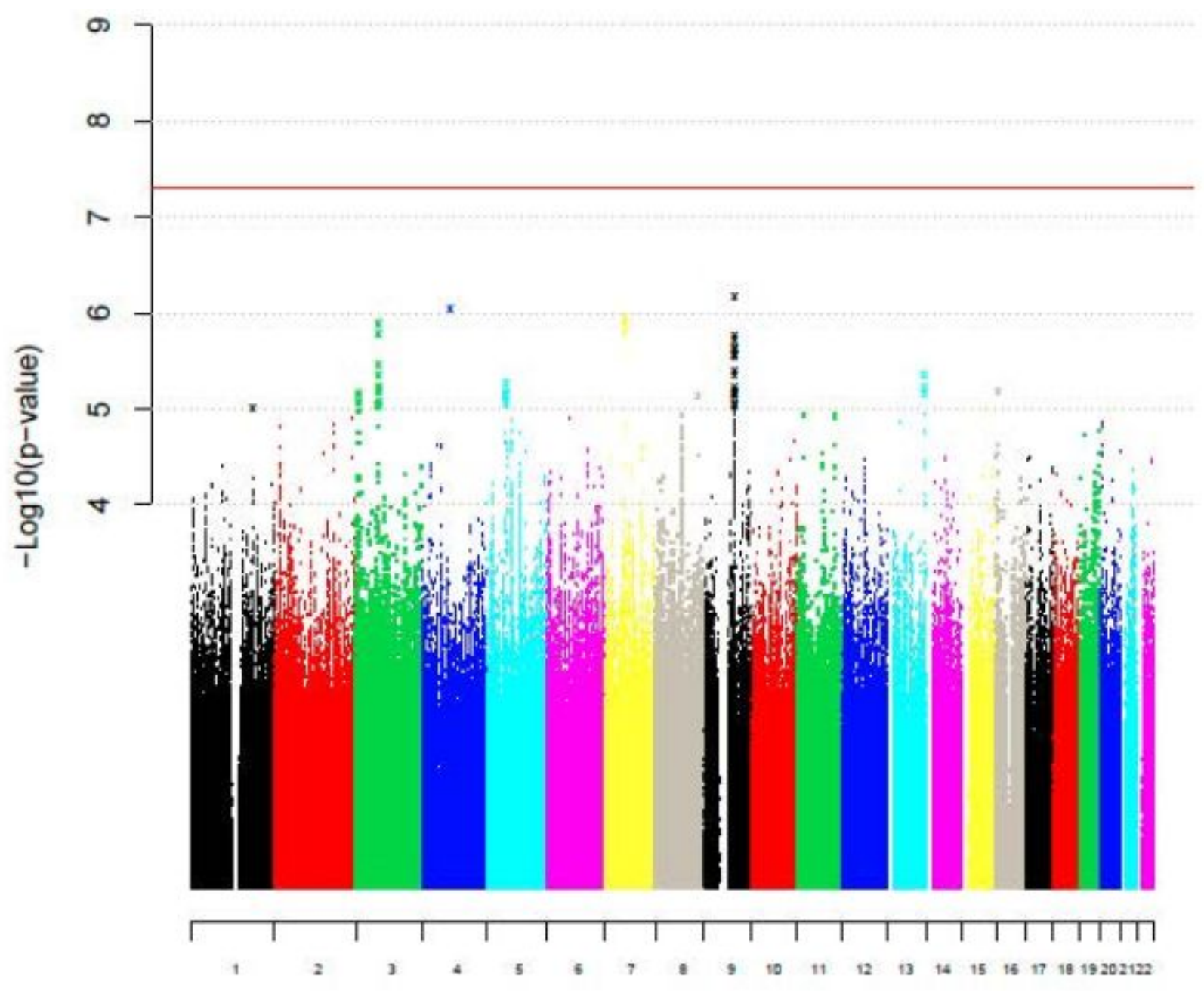

